\title{
False positive T-cell Cytotoxicity crossmatch results suggestive of autoantibodies in Korean Network for Organ Sharing crossmatch tests for deceased donor organ transplantation
}

\author{
Myoung Hee Park, Sohyun Kim, Eun Kyung Kwon, Eun Hee So, Kwang Woo Jeon, Sung Chang Yoon
}

KODA Laboratory, Korea Organ Donation Agency, Seoul, Korea

Background: For deceased donor organ transplantations in Korea, Korean Network for Organ Sharing (KONOS) mandates negative results of preliminary T-cell crossmatch (XM) for kidney and pancreas allocation. XM methods vary among different laboratories performing KONOS XMs and our laboratory is using complement-dependent cytotoxicity (CDC) (NIH and anti-human globulin $[\mathrm{AHG}]$ ) and flow cytometry methods. From our previous studies, we found autoantibodies usually show NIH+/AHG-/Flowresults, and less frequently $\mathrm{NIH}+/ \mathrm{AHG}+$ /Flow-results. We investigated the frequencies of false positive $\mathrm{T}$-cell $\mathrm{CDC}$ XM results suggestive of autoantibodies among KONOS XM results.

Methods: From January 2017 to September 2018, we performed KONOS XMs for T-cells by NIH, AHG, and flow cytometry methods for 463 deceased donors and 9,244 transplant candidates. We analyzed results suggestive of autoantibodies showing CDC+ $(\mathrm{NIH}+/ \mathrm{AHG}-$ or $\mathrm{NIH}+/ \mathrm{AHG}+)$ and Flow- (or weak with mean fluorescence intensity ratio <5.0) results.

Results: A total of $82(0.9 \%)$ of 9,244 XM cases revealed autoantibody features. Among these cases $64(78.0 \%)$ and $18(22.0 \%)$ showed $\mathrm{NIH}+/ \mathrm{AHG}-$ and $\mathrm{NIH+/AHG}$ + results, respectively, and 68 (82.9\%) showed Flow- results. Suggestive autoantibody positive rate varied among transplant candidates listed for different organs: kidney/kidney-pancreas $0.6 \%$ (44/7,218), lung 6.8\% $(23 / 338)$, and heart $1.3 \%(15 / 1,155)$. Some candidates showed positive results against two or more different donors: two kidney candidates, 2-4 donors; six lung candidates, 2-5 donors; three heart candidates, 2-4 donors. In two transplant candidates showing positive results against three or more donors, IgM autoantibody nature was confirmed by negative conversion of CDC XMs on dithiothreitol-treated sera.

Conclusions: Although less than $1 \%$ of the transplant candidates listed for deceased donor transplantation show false positive CDC XM results due to autoantibodies, they may be repeatedly excluded from transplantation. For accurate interpretation of autoantibody nature in these cases, difficulties are expected when performing CDC XM only and performing both CDC and flow XMs would be of help.

Corresponding author: Myoung Hee Park

E-mail: parkmhee@snu.ac.kr

(c) The Korean Society for Transplantation

This is an Open Access article distributed under the terms of the Creative Commons Attribution Non-Commercial License (http://creativecommons.org/licenses/by-nc/4.0/) which permits unrestricted non-commercial use, distribution, and reproduction in any medium, provided the original work is properly cited. 